
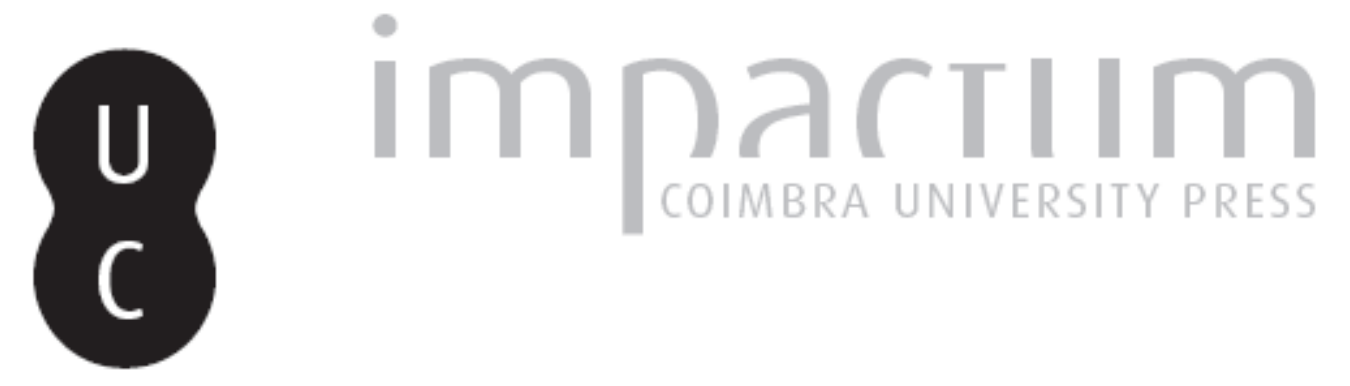

\title{
Sobre um eléctrico rápido
}

\section{Autor(es): $\quad$ Correia, Paulo Bebiano}

Publicado por: Editorial do Departamento de Arquitectura

URL persistente:

URI:http://hdl.handle.net/10316.2/37496

DOI:

DOI:http://dx.doi.org/10.14195/0874-6168_6.7-1_4

Accessed : $\quad$ 26-Apr-2023 09:04:04

A navegação consulta e descarregamento dos títulos inseridos nas Bibliotecas Digitais UC Digitalis, UC Pombalina e UC Impactum, pressupõem a aceitação plena e sem reservas dos Termos e Condições de Uso destas Bibliotecas Digitais, disponíveis em https://digitalis.uc.pt/pt-pt/termos.

Conforme exposto nos referidos Termos e Condições de Uso, o descarregamento de títulos de acesso restrito requer uma licença válida de autorização devendo o utilizador aceder ao(s) documento(s) a partir de um endereço de IP da instituição detentora da supramencionada licença.

Ao utilizador é apenas permitido o descarregamento para uso pessoal, pelo que o emprego do(s) título(s) descarregado(s) para outro fim, designadamente comercial, carece de autorização do respetivo autor ou editor da obra.

Na medida em que todas as obras da UC Digitalis se encontram protegidas pelo Código do Direito de Autor e Direitos Conexos e demais legislação aplicável, toda a cópia, parcial ou total, deste documento, nos casos em que é legalmente admitida, deverá conter ou fazer-se acompanhar por este aviso.

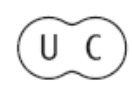




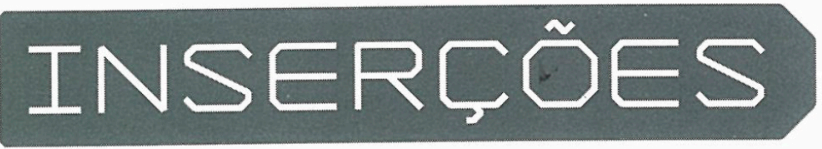

\title{
SEMINÁRIO INTERNACIONAL DE DESENHO URBANO
}

\author{
PT António Belém Lima \\ PT José Gigante \\ ISR Peter Keinan \\ ES Eduard Bru \\ PT Manuel Graça Dias + Egas José Vieira
}
BR MMBB
PT Eduardo Souto Moura
ES Emilio Tuñón + Luis Mansilla
PT Alexandre Alves Costa + Sergio Fernandez
PT Manuel Mateus + Francisco Mateus

\section{(COIMBRA COMO TERRITÓRIO)}

Walter Rossa

(PROJECTOS URBANOS EM COIMBRA )

José António Bandeirinha

(METRO LIGEIRO DE SUPERFICIE)

Gonçalo Byrne, José António Bandeirinha, Nuno Grande, Rui Lobo, Armando Rabaça

( SOBRE UM ELÉCTRICO RÁPIDO )

Paulo Bebiano Correia 


\section{METRO}

\section{SOBRE UM ELÉCTRICO RÁPIDO}

Paulo Bebiano Correia

\section{QUESTÕES VIVAS SOBRE UM "ELÉCTRICO RÁPIDO"}

\section{INTRODUÇÃO}

Os transportes públicos são um sector charneira da actividade municipal. É no sentido que quisermos dar à sua evolução ou, por antagonismo, ao seu desaparecimento, que se jogam muitas das opções fundamentais para a qualidade de vida dos cidadãos e para o próprio carácter da cidade.

Não podemos ambicionar reduzir a proliferação absurda dos meios de transporte individual, ou conseguir mais e melhores lugares para estacionar, ou poder andar nas praças e nos passeios sem tropeçar nos automóveis estacionados, se não possuirmos uma rede de transportes públicos rápida e eficaz, que garanta às pessoas suficiente maleabilidade de horários e possibilidades de acesso seguro, nas desloca-ções quotidianas da vida contemporânea.

Por outro lado, os transportes públicos simbolizam a colectivização de uma actividade. Merecem, também por essa razão, ser o alvo preferencial das preocupações dos cidadãos.

Por último, mas não menos importante, são partes significativas do carácter de uma cidade, e da urbanidade que the está subjacente. Em muitos casos, assumem-se mesmo como símbolos, ou, pelo menos, como valores emblemáticos dessas cidades, chegando mesmo a actuar como mais valia turística e cultural (como nos exemplos mais evidentes de Londres, Amsterdão, S. Francisco, e tantas outras).

Mas, no caso de Coimbra, este é, porventura também, o sector onde mais veementemente se sente a ausência de estratégias coordenadas de planeamento. Uma cidade que já possuiu uma das redes urbanas mais equilibradas do país, a única esperança que tem neste momento, para além das queixas generalizadas dos cidadãos que não vêem alternativas à utilização do automóvel individual, é a longínqua possibilidade de alțeração da linha da Lousã para um sistema de circulação mais ligeiro, tipo metro de superficie. Ora, sendo esta intenção realmente estruturante e decisiva, nada se sente e se sabe acerca da sua coordenação com a rede que ainda subsiste. Que articulação com as linhas existentes? Que linhas novas se intendem criar? Qual a possibilidade de vir a criar acessos mecânicos de encosta, integrados com os existentes? Que tipos de transporte para completar a linha de metro ligeiro? Enfim, que estratégia de desenvolvimento para os transportes públicos nesta cidade?

\section{CONCEITOS GERAIS SOBRE OS SERVIÇOS FERROVIÁRIOS LIGEIROS PÉRI-URBANOS}

\section{Da Multiplicidade das Definições à Ambiguidade Conceptual}

Quando se pretende servir uma zona péri-urbana ou "suburbana e regional", utilizando "material circulante ligeiro" sobre uma via ferroviária existente, é necessário clarificar, em função das diferentes situações, as noções por vezes mal definidas como as de "eléctrico rápido misto", "eléctrico rápido de interconexão", "eléctrico rápido de interoperabilidade", "eléctrico rápido híbrido", "eléctrico rápido comboio", "comboio eléctrico rápido", "eléctrico rápido de aglomeração" ou de "eléctrico rápido regional". Este vocabulário, em que a expressão "eléctrico rápido" pode ainda ser substituída por "metro ligeiro" traduz, sem dúvida, uma certa ambiguidade conceptual, em que os mesmos termos designam, de facto, segundo o contexto, diferentes tipos de material circulante, de sistemas de transporte, ou mesmo de organizações da oferta. 
Segundo (Chaine et Lhomet, 1997) podem definir-se três sistemas para um serviço ferroviário deste tipo:

O sistema "ferroviário ligeiro", (ii) o sistema "eléctrico rápido de interoperabilidade", (iii) e o sistema "eléctrico rápido de aglomeração".

o Sistema Ferroviário Ligeiro Sumariamente o "sistema ferroviário ligeiro" consiste em utilizar um material ferroviário de nova geração ( "design”, dimensões, etc....) para o serviço péri-urbano sem que exista interoperabilidade física com o sistema urbano. Neste caso é necessário assegurar uma boa coordenação modal entre este serviço e um serviço urbano de elevada qualidade. Se este sistema contribui para dar coerência à "bacia de vida" aproximando, através de uma ligação radial, os pólos secundários périurbanos da "cidade", a solução é, em termos teóricos, tanto mais satisfatória quanto menos penalizadora for a "ruptura de carga" (ou "transbordo") para os utentes do serviço.

o Sistema "Eléctrico Rápido de Interoperabilidade"Por sua vez, o sistema "eléctrico rápido de interoperabilidade" ${ }^{1}$, cujo material circulante é frequentemente designado por "tram-train" ou "eléctrico rápido - comboio", apoia-se na circulação deste tipo de veículos sobre uma via ferroviária existente que é fisicamente ligada às linhas ferroviárias de uma rede de "eléctricos rápidos urbanos". A interoperabilidade física, extremamente exigente na tecnologia empregue nos veículos bem como na sua operação, permite a ligação

\section{As Indefinições Para Serviço Péri-Urbano do "Ramal da Lousã"}

Quando, em face de uma situação concreta, se péri-equaciona a escolha de um dos três tipos possiveis de sistemas ferroviários ligeiros para substituir uma linha existente de $\mathrm{TER}^{2}$, como é o caso da parte suburbana e regional do “Ramal da Lousã”, a resposta correcta depende, genericamente, dos objectivos a atingir em termos de mobilidade e de organização do território, do contexto local mas também, e sobretudo, dos meios financeiros disponíveis.

A empresa MLM, Sa tem, nestes últimos anos, de forma mais ou menos explicita, vacilado entre um projecto de "eléctrico rápido de interoperabilidade" e um projecto de "eléctrico rápido de aglomeração".

Efectivamente, se por um lado a não separação dos tipos de veículos a utilizar ${ }^{3}$ parece apontar indubitavelmente, mas de forma manifestamente errática, para um sistema de "eléctrico rápido de aglomeração" as densidades popula- diametral, sem "ruptura de carga", entre os centros périurbanos e a cidade. Tal como no caso do "sistema ferroviário ligeiro" se a linha suburbana e regional for partilhada por veículos ferroviários clássicos, a recuperação urbana da "primeira coroa" na zona de passagem do eixo ferroviário è dificilmente conseguida.

"O Sistema de "Eléctrico Rápido de Aglomeração" Finalmente o sistema de "eléctrico rápido de aglomeração" consiste em dedicar inteiramente uma infra-estrutura ferroviária a um "eléctrico rápido" clássico. Neste caso, para a obtenção de uma maior velocidade de ponta nas grandes inter-estações, é necessária a adaptação da cadeia cinemática do "eléctrico rápido urbano". Para que um "eléctrico rápido de aglomeração" possa ser constituído, para além da existência de um elevado património ferroviário que possa ser desafectado dos serviços ferroviários clássicos, é sem dúvida necessária uma forte densidade populacional nas áreas de influência das estações deste sistema.

Sob o ponto de vista da estruturação da aglomeração às vantagens do sistema de "eléctrico rápido de interoperabilidade" este sistema adiciona ainda uma eventual possibilidade de recuperação urbana da "primeira coroa".

São exemplos deste tipo de sistema o "eléctrico rápido" de Val-de-Seine na Região Parisiense o "Metro-Link" Bury-Altrincham de Manchester ou o "Metro do Porto".

cionais das zonas suburbanas e regionais a servir e a estrutura urbana de Coimbra não permitem propor de forma lúcida, mas já arrojada e talvez ingénua, mais do que um sistema de "eléctrico rápido de interoperabilidade".

Se a validação de um esquema de princípio de um "eléctrico rápido urbano" para Coimbra foi conseguida após seis anos de "aprofundados estudos" feitos pela MLM Sa., conviria não perder a oportunidade histórica de concretizar um sistema deste tipo por incapacidade de concepção da parte suburbana e regional do "Ramal da Lousã".
1 Por interoperabilidade deve entender-se

"interoperabilidade fisica" tal como tol definida pelo GDR "Résaux".

"eléctrico rápida" pode ser substituida, no contexto deste documento. por "Metro Ligeiro". 2 Transporte Expresso
Regional

3 Por razões que se prender com os custos do material circulante 


\section{SINTESE DOS CONCEITOS SUBJACENTES \\ À REALIZAÇÃO DE UM SISTEMA \\ DE “ELÉCTRICO RÁPIDO DE INTEROPERABILIDADE”}

Para avaliar a pertinência de servir, a curto prazo, a parte suburbana e regional do "Ramal da Lousã" com um "eléctrico rápido de interoperabilidade" analisaram-se, ainda que de forma sumária, as características os objectivos e os pré-requisitos deste tipo de sistema. (Ver anexo I). Nesta secção é feita uma breve síntese dos conceitos mais relevantes.

\section{Dos Projectos e das Realizações}

No que diz respeito aos projectos e às realizações:

_Se se exceptuar o Caso de Sarrebruck - Sarreguemines, é sistemática a separação dos projectos técnicos e respectivas realizações relativamente às linhas de rede urbana de "eléctrico rápido urbano" - concretizadas sempre em primeiro lugar - e ao sistema de "eléctrico rápido de interoperabilidade".

_Nas realizações dos projectos deste tipo de sistema é imprescindível o "know-how" do operador ferroviário nacional que poderá posteriormente ser transferido para um operador local (Caso da DB nos projectos alemães e da SNCF nos projectos franceses).

\section{Da Exploração}

São condições essenciais para o sucesso de um sistema de "eléctrico rápido de interoperabilidade":

_Evitar uma ruptura de carga quando esta é fortemente penalizante para utentes da linhas suburbanas e regionais.

_Garantir uma redução dos tempos de percurso, ainda que servindo um maior número de estações.

_Responder ao aumento da procura, que pode ser 3 a 4 vezes superior relativamente à procura previamente existente, com um aumento da oferta adequado.

São ainda condições relevantes para a adopção de um sistema deste tipo:

_Permitir estabelecer uma ligação diametral entre os pólos péri-urbanos e a cidade.

_Não interromper o serviço suburbano e regional previamente existente por tempo demasiado longo.

\section{Dos Custos de Investimento}

Relativamente aos custos de investimento para a constituição de um sistema de "eléctrico rápido de interoperabilidade":

_O custo do material circulante é relativamente elevado e cerca de $50 \%$ superior ao de um "eléctrico rápido urbano" (cujo valor actual ronda os 450.000 contos para uma composição de 30 metros). 0 custo actual de um tram-train situa-se entre os 600.000 e os 700.000 contos.

_É imprescindível a existência de uma via ferroviária de gabarito estandardizado e electrificada (15 kV ou $25 \mathrm{kV}$ ) para que os custos de investimento de um sistema deste tipo possam ser aceitáveis relativamente aos ganhos expectáveis, nomeadamente em termos de aumento da procura e de redução dos custos de exploração.

\section{Da Tecnologia}

_Cerca de dez anos após o início da exploração do primeiro de "eléctrico rápido de interoperabilidade" este sistema pode ser considerado extremamente exigente sob o ponto de vista tecnológico.

_A existência de um único veículo testado em exploração comercial e simultaneamente "transponível" para outros contextos ("tram-train" de Sarrebruck-Sarreguemines da Bombardier transport) aconselha, seguramente, uma certa prudência na realização de um sistema de "eléctrico rápido de interoperabilidade". Deve notar-se que neste momento nem a Siemens nem a Alsthom dispõem de veículos testados para equipar este tipo de sistemas.

_A entrada em circulação, quase que simultaneamente, de "eléctricos rápidos urbanos" e de "eléctricos rápidos de interoperabilidade" aumenta fortemente os riscos de derrapagem tecnológica do sistema. 


\section{SOBRE A ADEQUAÇÃO DE UM SISTEMA DE “ELÉCTRICO RÁPIDO DE INTEROPERABILIDADE” À PARTE SUBURBANA E REGIONAL. DO “RAMAL DA LOUSÃ”}

\section{O Serviço de Transporte Prestado}

A Velocidade Comercial e a Oferta Das declarações públicas proferidas pela MLM Sa., pode depreender-se que não há redução substancial dos tempos de percurso no serviço suburbano e regional. Se este facto põe indubitavelmente em causa a adopção do sistema proposto, não é menos preocupante o facto de parecer não haver uma resposta adequada, em termos de oferta, aos aumentos de procura que justificariam a realização de um "eléctrico rápido de interoperabilidade".

\section{A Interligação da Circulação Urbana com a Circulação} Suburbana e Regional A estes factos acresce uma dificuldade de exploração do sistema, que a empresa MLM, Sa. parece omitir: a exploração em zona urbana de uma rede de eléctricos rápidos com uma topologia em "estrela de três ramos", não permite intercalar com facilidade composições com origem na zona suburbana ou regional. Contudo, a MLM, Sa. propõe que as composições provenientes das zonas suburbanas e regionais, sejam intercaladas quer nas ligação à Estação Coimbra B, quer nas ligações com destino aos H.U.C.

Mas, efectivamente, toda a elaboração de "diagramas de marcha" que pretenda ser mais do que um mero exercício de estilo, deve ter em conta que, no caso de Coimbra, a entrada de composições suburbanas e regionais na parte urbana da rede de eléctrico rápido estaria, na prática, sujeita a um "tempo de regulação" que, do ponto de vista do operador se traduz por uma redução efectiva das velocidades de rotação e, do ponto de vista dos utentes por um acréscimo do tempo de espera na estação de entrada/ /saída do percurso suburbano. Este tempo de espera adicional, ainda que em melhores condições de conforto para os passageiros sentados, traduz-se na prática por algo semelhante a uma ruptura de carga entre o suburbano e regional e o urbano.

A Adaptação da Linha Existente Bem mais preocupante é, no entanto, a interrupção do serviço suburbano e regional por um período de cerca de dois a dois anos e meio, para armar a integralidade da plataforma do Ramal da Lousã com bitola estandardizada e para proceder à sua electrificação. As consequências da mais do que provável erosão da procura existente, ainda que quantitativamente imprevi- síveis, não serão, seguramente, de negligenciar. A oferta de um transporte público rodoviário de substituição, provavelmente de menor qualidade, pode alterar, de forma dificilmente reversivel, os hábitos modais dos utentes do actual "Ramal da Lousã".

Pode concluir-se que, sobe o ponto de vista do serviço prestado, a proposta da MLM Sa. fere uma grande parte dos princípios subjacentes à realização e à exploração de um sistema de "eléctrico rápido de interoperabilidade".

\section{Os Custos do Sistema Face aos Objectivos Propostos}

Se o novo sistema, tal como é proposto pela MLM Sa., parece não dar uma resposta totalmente convincente, que se traduziria por uma melhoria substancial da oferta face à do serviço actual, convém ainda questionar os custos do novo sistema proposto.

Mais uma vez a MLM Sa. parece não compreender que, em termos de rendibilidade do investimento, mesmo que no sentido não estritamente "economicista" do termo, é condição sine qua non para a realização de um sistema de "eléctrico rápido de interoperabilidade" a existência prévia de uma linha ferroviária de gabarito estandardizado electrificada a 15 ou $25 \mathrm{kV}$.

Se, como se tem verificado internacionalmente, os custos de investimento na realização de um sistema deste tipo são já relativamente elevados, no caso do "Ramal da Lousã" a necessidade de rearmar toda a plataforma em bitola estandardizada e de electrificar a via implicará, seguramente, custos adicionais muito significativos.

Ainda que de forma aproximada, poder-se-à estimar que os custos de investimento da parte suburbana e regional do Ramal da Lousã, entre a estação das Carvalhosas e Serpins, se situem entre os 20 e os 30 milhões de contos.

Dado o estado de avanço do anteprojecto os custos de investimento estimados pela MLM Sa. de cerca 45 milhões de contos para o "eléctrico rápido urbano" e para o sistema de "eléctrico rápido de interoperabilidade" podem ser realistas "para a melhor das hipóteses" ou se "tudo correr bem". É no entanto de temer que, com o aprofundamento dos estudos e em fase de projecto técnico - feito pelos concorrentes num curto espaço de tempo - as "transferências de risco" para o construtor e para o operador, propostas pela MLM Sa, se traduzam, na prática, por um acréscimo de custos que, por ser incomportável, venha a por em causa todo sistema. 


\section{Os Riscos Tecnológicos}

Tal como foi referido, o sistema de "eléctrico rápido de interoperabilidade" para além de ser extremamente exigente tecnologicamente está ainda numa fase de desenvolvimento relativamente insipiente. Propor, tal como a MLM Sa. o fez, que veículos de interoperabilidade circulem num traçado urbano complexo, nomeadamente no troço "Arnado - HUC" revela, no mínimo, um aventureirismo tecnológico que deve ser evitado.

Pensar, como a MLM Sa., que os eventuais problemas tecnológicos se resolvem com a transferência dos riscos para o construtor e para operador é também manifesta ingenuidade. De facto, a prática tem demonstrado que, quando há derrapagens tecnológicas de sistemas de "eléctricos rápidos" em que se tenha incorporado uma forte dose de "inovação tecnológica", a factura é na sua maior parte paga pela cidade onde o sistema foi construído, tanto ao nivel das repercussões na sua imagem internacional como também ao dos problemas inerentes ao disfuncionamento, por períodos mais ou menos longos, de todo o seu sistema de transportes.

\section{BREVES CONSIDERAÇÕES \\ SOBRE A ADEQUAC̣ÃO \\ DE UM SISTEMA DE “ELÉCTRICO RÁPIDO \\ DE AGLOMERAÇÃO" A COIMBRA}

Uma análise da adequação de um sistema de "eléctrico rápido de aglomeração" para a cidade de Coimbra e para a parte suburbana e regional do "Ramal da Lousã", suscitaria questões que levariam a conclusões ainda mais severas e que concluiriam pela sua não viabilidade.

Desde a qualidade do serviço de transporte prestado em zona suburbana e regional, aos custos do sistema e em particular aos custos de exploração ou aos riscos tecnológicos, muitas das questões levantadas a propósito do sistema de "eléctrico rápido de interoperabilidade" teriam a mesma resposta. Outras questões de natureza técnica ou económica seriam de natureza a rejeitar liminarmente a adopção de um tal sistema.

\section{Conclusões}

Pode concluir-se que, a curto ou médio prazo, um sistema de "eléctrico rápido de interoperabilidade" é provavelmente desadequado ao serviço da parte suburbana e regional do "Ramal da Lousã”.

Tendo sempre presentes as dificuldades levantadas pelas particularidades da rede ferroviária nacional, a viabilidade de um tal sistema poderia no entanto ser convenientemente estudada, a mais longo prazo, e em particular no caso da constituição de uma linha diametral leste-oeste.

\section{AS QUESTÕES ESSENCIAIS}

O lançamento numa só fase de um concurso que contemple simultaneamente um "eléctrico rápido urbano" e um "eléctrico rápido de interoperabilidade" não pode deixar de ser motivo de preocupações e interrogações.

Justificar-se-à numa primeira fase servir a parte suburbana e regional do "Ramal da Lousã" com um sistema de "eléctrico rápido de interoperabilidade", sob o risco de inviabilizar tecnico-economicamente todo o projecto?

Ou, devem separar-se os concursos contemplando-se, numa primeira fase, a realização de um "eléctrico rápido urbano" deixando em aberto um eventual concurso subsequente para o suburbano e regional?

A resposta, em particular à segunda questão, contém inplicitamente uma outra. Como articular, neste caso, o "elécrtico rápido urbano" com o serviço suburbano e regional do "Ramal da Lousã"? 


\section{A ARTICULAÇÃO DO ELÉCTRICO RÁPIDO URBANO COM O SERVIÇO SUBURBANO E REGIONAL DO "RAMAL DA LOUSÃ"}

Para articular o serviço existente no "Ramal da Lousã" com o "eléctrico rápido urbano" é necessário responder a duas questões:

1) $\grave{A}$ da necessidade das composiçōes que hoje servem a parte suburbana e regional, passarem para os serviços de manutenção da CP utilizando plataforma do "eléctrico rápido urbano" no eixo S. José-Solum. Esta passagem deverá, evidentemente, efectuar-se fora das horas de serviço do "eléctrico rápido urbano".

2) À da criação de uma paragem, terminus do serviço suburbano e regional e ponto de "ruptura de carga" (ou transbordo) entre este serviço e a rede de transportes públicos urbanos de Coimbra (incluindo o "eléctrico rápido" urbano).

A resposta à primeira questão passa, pela possibilidade de inclusão de um par adicional de carris ou, quando tal for possível de um "terceiro carril", formando-se assim uma via de "bitola ibérica" na plataforma do "eléctrico rápido urbano" durante a execução do troço Solum-Sul - Estação de Coimbra B.

Tecnicamente, a realização de plataformas de "eléctrico rápido urbano" em laje contínua de betão, evitando a colocação de "travessas", é actualmente tecnicamente possível, testada, sem sobrecustos e de execução mais rápida. A inclusão de um par adicional de carris, sobre uma plataforma em laje contínua, poderá assim ser viável, sem custos adicionais excessivos. Se os compararmos com os custos inerentes à proposta de "rearmação" integral em bitola estandardizada da parte suburbana e regional do "Ramal da Lousã", estes custos adicionais revelar-se-ão, muito provavelmente, irrisórios. A uma maior velocidade de execução da referida plataforma urbana, de cerca de 5 a $6 \mathrm{~km}$, corresponderia um tempo de interrupção relativamente curto do serviço suburbano.
Acresce que, sem se pretender fazer um faseamento das obras de construção do "eléctrico rápido urbano", a execução da plataforma e a circulação do "eléctrico rápido" no referido eixo, antes da conclusão do denominado "troço urbano central", teria duas vantagens inquestionáveis:

A primeira é a da utilização deste eixo para apoiar a estruturação das redes de transporte públicos rodoviários de superfície durante a execução do "ramal urbano central".

A segunda, de ordem mais política, está ligada aos “incómodos ou transtornos" para a Cidade, causados pela execução do eixo "Arnado - HUC". Sendo o troço "Coimbra B - Solum Sul" de inserção muito menos "dura" a sua entrada em funcionamento, ao prefigurar o conjunto do sistema, contribui de forma importante para uma melhor aceitação, por parte dos cidadãos, dos trabalhos de execução do "troço urbano central".

A questão da "ruptura de carga", foi analisada em 1996. Desde que a "ruptura de carga" seja localizada onde então foi prevista, esta torna-se relativamente pouco penalizante para os utentes do suburbano e regional. De facto com apenas um trans-bordo, será possível aos referidos utentes acederem, em transporte público, a uma grande parte do território urbano. Note-se que entretanto, se criou através da "Ponte Europa", a possibilidade da ampliaçāo da acessibilidade directa a partir deste nó à margem esquerda por ligação em linha de autocarros.

Se, com a concretização do "eléctrico rápido urbano", os utentes do "Ramal da Lousã" estarão entre os primeiros beneficiados, por se poderem deslocar em Coimbra numa rede de transporte público muito mais eficiente, não é menos verdade que o sistema criado seria, a médio longo prazo, garante da perenidade do "Ramal da Lousã".

Por outro lado esta proposta de articulação do "eléctrico rápido urbano" com o serviço suburbano e regional do "Ramal da Lousã", ao ser concretizada, deixaria em aberto um vasto leque de hipóteses de reestruturação do ramal, que mereceria estudos sérios e aprofundados. 
O eléctrico Rápido de Interoperabilidade:

Tendências Europeias e Custos de Sistema
Karlsruhe, 1992 Tecnicamente, o conceito de "eléctrico rápido de interoperab́ilidade" é desenvolvido na Alemanha. (Quidort, 1992). O objectivo era de interconectar uma linha ferroviária clássica de cerca de $25 \mathrm{Km}$ de comprimento, em via única, electrificada a $15 \mathrm{KV}$ CA e explorada pela Deutsche Bundesbhun, que ligava estação central da cidade de Karlsruhe a Breten, à rede de eléctricos rápidos urbanos da cidade, operada pela Verkehrsbertieb, por forma a evitar uma ruptura de carga considerada demasiado penalizante para os utentes do serviço ferroviário péri-urbano na ligação Bretten - Karlsruhe.

Os estudos começaram a desenvolver-se em 1986 e, entre junho de 1991 e julho de 1992, foi testado com sucesso um veículo bi-corrente capaz de operar a $15 \mathrm{kV}$ ca em linha ferroviária clássica e a 550 V cc na rede urbana. Podendo atingir velocidades de ponta da ordem dos $100 \mathrm{~km} / \mathrm{h}$ e preparado para resistir ao choque em circulação ferroviária clássica o veículo concebido era de plataforma elevada e de gabarito compatível com o dos "eléctricos rápidos urbanos". (Sutter, 1994).

A exploração comercial entre Karlsruhe e Bretten, que serve na linha ferroviária clássica 16 estações de quais de $58 \mathrm{~cm}$ de altura, teve início em fins de Setembro de 1992 e traduziu-se por um aumento substancial da procura. A velocidade comercial fora da zona urbana é de $60 \mathrm{~km} / \mathrm{h}$.

Sarrebruck-Sarreguemines, 19970 serviço que, nos finais de Outubro de 1997 ligou a cidade de Sarrebruck na Alemanha a Sarreguemines em França é, em alguns aspectos, idêntico ao de Karlsruhe.

A constituição do sistema foi feita apoiando-se num serviço ferroviário existente de via dupla com electrificação ferroviária clássica de $15 \mathrm{kV}$ ca (se se exceptuar o troço de via única de cerca de $1 \mathrm{~km}$ que liga a fronteira a Sarreguemines). A construção de cerca de $5 \mathrm{Km}$ adicionais de via, parte dos quais em meio urbano e electrificados a $750 \mathrm{~V}$ cc, permitiu dotar Sarrebruck de "eléctrico rápido de interoperabilidade". Com um comprimento total de cerca de 19 Km e 21 estações a linha é explorada por veículos bi-corrente, de plataforma parcialmente rebaixada. Em 1997, a velocidade comercial média (em zona urbana e péri-urbana) era de $43 \mathrm{~km} / \mathrm{h}$.

Numa segunda fase o sistema deveria ser ampliado passando a ter um comprimento de $44 \mathrm{Km}$ de vias, 15 das quais seriam
Os Projectos Franceses de "Eléctrico Rápido de Interoperabilidade" São inúmeras as cidades francesas que nestes últimos anos têm desenvolvido projectos de sistemas de "eléctrico rápido de interoperabilidade" (Paris, Lyon, Lille, Estrasburgo, Grenoble, Nantes, Moulhouse, St Étienne,...). Os mais avançados são, porventura, os da linha entre Aulnay-sous-Bois e Bondy na "Região Parisiense", o da linha de "Moulhouse", os das linhas de Estrasburgo, de Lyon e de St. Etiénne.

De facto em 2001 a SNCF examinou as condições de um caderno de encargos para o fornecimento de 79 composições de tram-train das quais 15 "confirmadas" para a linha ferroviária entre Aulnay-sous-Bois e Bondy, que deverá entrar em funcionamento em meados de 2005, e 64 opcionais para os projectos de Estrasburgo, Moulhouse, St Étienne e Lyon (Chlastacz, 2001).

A linha entre Aulnay-sous-Bois e Bondy será provavelmente o primeiro teste do sistema "eléctrico rápido de interoperabilidade" em França e, desta experimentação, serão seguramente retirados os ensinamentos para os projectos mais avançados de algumas cidades francesas.

Em França, todos os projectos de um sistema de "eléctrico rápido de interoperabilidade" desenvolvidos têm tido dois denominadores comuns. Por um lado todas estas cidades para as quais existem projectos deste tipo de sistema têm - ou serão previamente dotadas, tal como em Moulhouse de redes de transporte público urbano ferroviário de grande qualidade. ("eléctrico rápido urbano", "metro automático ligeiro" (VAL), "metro pesado"). Por outro lado os projectos e os concursos para a construção de um "eléctrico rápido urbano" têm sido, sistematicamente, separados dos projectos e dos concursos para um sistema de "eléctrico rápido de interoperabilidade"

\section{Por outro lado os projectos e os} concursos oara a construção da linha um 'eléctrico rápido urbano' têm sido, sistematicamente, separados dos projectos e concursos para um sistema de 'eléctrico rápido de interoperabilidade'. 


\section{Os Custos do Sistema "Eléctrico Rápido de Interoperabilidade"}

As potencialidades de transposição do sistema de "eléctrico rápido de interoperabilidade" para as redes francesas bem como as estimativas dos os custos de investimento e de exploração que Ihes estariam associados foram analisados por Chaigneau et Pécheur, 1997. Para o caso de uma infra-estrutura ferroviária existente (linha de bitola estandardizada e electrificada) o custo de investimento cifar-se-ia, a preços de 1997, entre os 20 e 35 milhões de FF por $\mathrm{Km}$ (cerca de 0.6 milhões a 1.05 milhões de contos por $\mathrm{km}$ ) Nestes valores está incluído o custo do material circulante, estimado em de cerca de 15 milhões de FF por composição (cerca de 450 mil contos) ou seja, $50 \%$ superior ao do "eléctrico rápido urbano" (o preço do $\mathrm{TFS}^{4}$ era, à data, de cerca de 10 milhões de FF ou seja, cerca de 300.000 contos)

0 custo de exploração foi avaliado em cerca de 35 FF/ veículo $\mathrm{x} \mathrm{Km}$. (equivalente ao da exploração de um "eléctrico rápido urbano").
Na sua segunda fase o custo do sistema de "eléctrico rápido de interoperabilidade" de Sarrebruck, seria a preços de 1998, segundo (Keudel, 1998) de cerca 540 Milhões de DM. (cerca de 54 milhões de contos) ou 12.3 milhões de DM por $\mathrm{km} \mathrm{O}$ custo de cada unidade de material circulante cifrava-se em cerca de 600.000 contos.

A adjudicação do fornecimento à SNCF de 35 composições de tram-train do modelo "Avanto" foi feita à Siemens em Julho de 2002 por 122 milhões de euros (cerca 3.49 milhões de euros ou 700 mil contos por composição). A linha entre Aulnay-sous-Bois e Bondy na RIF será equipada por 15 destas composições de piso rebaixado (sendo a compra das restantes 30 opcional). Prevê-se assim que em meados 2005 em Paris, a SNCF possa explorar pela primeira vez um sistema de "eléctrico rápido de interoperabilidade" tal como o tinha feito, 13 anos antes, a Alemanha em Karlsruhe.(Chlastacz, 2002;Trans flash, 2002).

\section{BIBLIOGRAFIA}

CHAIGNEAU, Éllsabeth O PÉcheUR, Paseale

(1997) Quand le tramway sort de la ville: des nouvelles réponses ferroviaires à la desserte des périphéries d'agglomération. In: Transports Urbains. octobre-décembre 1997, n 97. pp.15-24

CHAINE, Hervó et LHOMET, Étienne.

(1997) Le rôle des tramways "d'interconnexion" dans l'amménagement des térritoires. In: Transports Urbains. 1997, octobre-décembre, $n^{\circ}$ 97, pp. 25-30.

CHLASTACZ, Michol.

(2001) La SNCF lance un appel d'offre pour 79 rames de tram-trains. In: Transport Public. février 2001, n' 1000, p.8.

(2002) Tram-train: la SNCF choisit "Avanto" de Siemens. In: Transport Public. mal 2002 n० 1014 , p.9.
KEUDEL, Walter.

(1998) La Saarbahn - Le nouveau système de transport urbain et régional sur vole ferrée de la région de Sarrebruk. In: Transport Public International. juillet 1998, $n^{\circ} 4$ - vol. 47 , pp. $25-31$

QUIDORT, Michol

(1992). Les lignes ferroviaires régionales a I'heure des choix - Experiences nouvelles: regard sur trois voisins européens (Colloque FNAUT de Tours (octobre 1992)). In: Transports Urbains, octobre décembre $1992, n^{\circ} 77$. D. $27-30$.

SUTTER, Alain

(1994) Interconnexion physique entre métro leger et réseau ferré national a Karisruhe... et ailleurs. In: Transports Urbalns. octobre-décembre 1994, n*85, pp.19-22.

TRANSFLASH

(2002) La SNCF commande 35 tram-trains pour la ligne dite des "Coquetiers" entre Aluny. sous-Bois et Bondy en ile-de-France. In: TransFlash., septembre 2002, $n^{\circ} 274, p .2$. 